\title{
Images and Spectral Properties of Two Component Advective Flows Around Black Holes: Effects of Photon Bending
}

\author{
Arka Chatterjee ${ }^{\star 1}$, Sandip K. Chakrabarti $\dagger^{2,1}$ and Himadri Ghosh $\ddagger^{3}$ \\ ${ }^{1}$ Indian Centre for Space Physics, Chalantika 43, Garia Station Rd., Kolkata, 700084, India \\ ${ }^{2}$ S. N. Bose National Centre for Basic Sciences, Salt Lake, Kolkata, 700098, India \\ ${ }^{3}$ Heritage Institute of Technology, Kolkata, 70010\%, India
}

\begin{abstract}
Two component advective flow (TCAF) successfully explains spectral and timing properties of black hole candidates. We study the nature of photon trajectories in the vicinity of a Schwarzschild black hole and incorporate this in predicting images of TCAF with a black hole at the Centre. We also compute the emitted spectra. We employ a Monte-Carlo simulation technique to achieve our goal. For accurate prediction of the image and the spectra, null trajectories are generated without constraining the motion to any specific plane. Red shift, bolometric flux and corresponding temperature have been calculated with appropriate relativistic consideration. The centrifugal barrier dominated boundary layer or CENBOL near the inner region of the disk which acts as the Compton cloud is appropriately modelled as a thick accretion disk in Schwarzschild geometry for the purpose of imaging and computing spectra. The variations of spectra and image with physical parameters such as the accretion rate $\left(\dot{m}_{d}\right)$ and inclination angle are presented. We show that the gravitational bending effects of photons do change the spectral shape to some extent.
\end{abstract}

Key words: black hole physics - accretion, accretion discs - relativistic processes - radiative transfer

* arka@csp.res.in

$\ddagger$ himadri.ghosh@heritageit.edu 


\section{Arka Chatterjee, Sandip K. Chakrabarti \&3 Himadri Ghosh}

\section{INTRODUCTION}

Identification of any black hole candidate (hereafter BHC) involves in observing radiations from matter accreted on it. Radiations give out information on spectral and temporal properties in X-rays, UV or optical band. In near future, with the advent of high-resolution imaging devices, imaging of such BHCs with matter all around will be possible. It is therefore essential to follow paths of photons emitted from hydrodynamically correct matter distribution with successful spectral features very accurately till they reach observer's image plane. This would enable us to obtain the spectra and images from different inclination angles. In this paper, we achieve this by taking a two component advective flow (TCAF) solution based on rigorous theory of transonic astrophysical flows (Chakrabarti, 1990) and computing emitted radiation by a Monte-Carlo simulation.

Bending of photons in a gravitational field is a natural consequence of general theory of relativity. In our context, photons emitted from the TCAF will be deviated by the strong gravitation field created by the black hole. Deviations of photons depend on the space-time metric and its impact parameter. Due to focusing effects, softer photons from the Keplerian component may be forced to intercept hot electrons in the post-shock region of the low-angular momentum component located close to the horizon modifying the spectrum. Theoretical work on imaging of emitting matter surrounding a black hole started in 1970s. The first step towards this emitter-to-observer ray tracing was attempted by Cunningham \& Bardeen (1973). Isoradial curves were drawn for a rotating black hole at some specified inclination angle. Pineault \& Roeder (1977) repeated this work with the variation of spin parameter. A complete image of a black hole surrounded by rotating matter was first drawn by Luminet in 1979. Idealized disk particles with differential rotational velocity were kept on the equatorial plane to construct a Keplerian disk. Mass of the disk was considered to be negligible as compared to the mass of the black hole. Emission of photon was assumed to be isotropic in nature. Bolometric flux of the Keplerian disk and red-shift of photons were included in the image. The basic process of generating an image was considered to be a boundary value problem, connecting a disk and an observer. In that way, the transfer function using elliptic integral is a time saver. Fukue \& Yokoyama (1988) produced a color photograph of a multi-wavelength study of the disk. They also studied flux variation in an eclipsing binary. Karas et al. (1992) developed a catalogue of photon trajectories with variable inclination angle and spin. Viergutz (1993) first attempted emitter-to-observer problem 
in the Kerr metric by generalizing the transfer function with rotating geometry. Until this work, images were drawn using semi-analytical methods, by computing a relation between the impact parameter $(b)$, polar angle $(\alpha)$ on observer plane and observer's polar angle $\left(\theta_{\circ}\right)$. Marck (1996) integrated each trajectory of photons to generate an image for the first time. We have followed this approach in this paper. However, instead of using first order differential equations, we traced the ray using three dimensional second order equations to draw the complete photon trajectory. This gives us the freedom to use analytically established as well as time dependent three dimensional disks emitting photons with all known phase-space coordinates (initial position and momentum of photons) as a starting configuration.

In twenty-first century, the use of X-ray polarimetry flourished rapidly. With that, Bromley et al. (2001) added polarization while drawing the disk image. Armitage and Reynolds (2003) performed magneto-hydrodynamics (MHD) on Keplerian disk and provided temporal variations of a Keplerian disk. Müller \& Frauendiener (2011) developed Figures using the same transfer functions. 'GYOTO' (Vincent et. al. 2011) is an open software available for imaging and trajectory calculations. More recently, 'YNOGK' (Yang \& Wang 2013), another publicly available code for Kerr space time was published. However, the present literature is mostly dominated by the images of a most ideal disk configuration, namely, a Keplerian disk, which unfortunately does not describe all the spectral states accurately. More recently, Younsi et al. (2012) published images containing relativistic thick disks for different inclinations and spin parameter with variation of gas pressure to radiation pressure ratios. However, so far, study of disk configurations, such as TCAF (Chakrabarti 1990; Chakrabarti \& Titarchuk, 1995; hereafter CT95), in different spectral states have been missing.

Since a black hole is very compact, the resolving power of a telescope must be very high to capture images close to the horizon. So, imaging by the so-called Event Horizon Telescope concentrates on nearby relatively massive black holes such as those present at the centre of our galaxy, namely, Sgr A* (Johnson et al. 2015). However, so far, no major attempt has been made to study the difference in images when the BHC is in the soft state and in the hard state, i.e., changes in the configuration of the disk itself. Part of the reason is that the model builders of spectral properties were keeping a Keplerian disk as the basis of all such spectral states and only the randomly placed Compton clouds (clouds of hot electrons) would change its properties. The hot cloud is required for inverse Compton scattering (Sunyaev \& Titarchuk, 1985). A concrete understanding of where the Compton cloud is really located and how its geometry vis-a-vis the Keplerian disk should change with 


\section{Arka Chatterjee, Sandip K. Chakrabarti 83 Himadri Ghosh}

spectral states came about only after successful application of the transonic flow solution in the context of black hole accretion by CT95. This so-called two component advective flow (TCAF) solution is regularly applied to explain spectral evolutions of numerous outbursting BHCs (see, Debnath et al. 2014; Mondal et al. 2014; Jana et al. 2015; Molla et al. 2016; Debjit et al. 2016) and actual physical parameter such as the accretion rates in the two components, the compression ratio of the centrifugally supported shock structure and the size of the Compton cloud itself were derived. According to CT95, state transitions are due to changes in accretion rates, only the time scale of such changes varies with the mass of the black hole. Higher accretion rates in the Keplerian component relative to the advective component cools the hot electrons in the Compton cloud shrinking it to a small size resulting in a softer state. In the opposite case of higher advective flow rate, Compton cloud (postshock region) remains hotter and a hard state is produced with a truncated Keplerian disk with its inner edge coinciding with the Compton cloud boundary or the shock location. So, geometry of the flow drastically changes with the spectral state. This is not common in massive black holes but there are certainly active galaxies with both classes of spectra. In stellar mass black holes, this change of geometry is common and quicker. For instance, in GRS $1915+105$, there are more than dozens of variability classes and all these are physically distinguished by the system geometry governed by the size of the Compton cloud and the truncated Keplerian component (Pal \& Chakrabarti, 2011, 2013).

One of the important results by an earlier numerical simulations by Molteni et al. (1994) is that the post-shock region (otherwise called the CENtrifugal pressure supported BOundary Layer or CENBOL, used as the Compton cloud in TCAF of CT95) of a transonic flow has most of the characteristics of a thick accretion torus (Abramowicz et al., 1978 and Kozlowski et al., 1978; Paczyński \& Wiita, 1980; Begelman. Blandford \& Rees, 1982; Chakrabarti, 1985, hereafter C85), except that CENBOL also has a radial motion and thus more accurate than the thick disk models of early eighties mentioned above. In an attempt to have a stationary image we require a theoretical solution for the CENBOL. We therefore use the fully general relativistic thick torus solution of Chakrabarti (1985) as the CENBOL along with a truncated Keplerian disk to have a theoretical model of a two component advective flow. To have different spectral states we change the accretion rates of these two components. To generate the spectrum from it, we employ a Monte-Carlo simulation code where soft photons from the truncated Keplerian component are allowed to (inverse) Compton scatter from the relativistic Maxwellian electrons inside the CENBOL. Monte-Carlo process (Pozdnyakov et al., 
1983) provides one of the best techniques to generate the complete spectrum. The power-law component in the observed spectrum of black hole candidates is the result of repeated inverse Comptonization (Sunyaev \& Titarchuk, 1985) of the soft photons. Thus, combining thermal multi-colour black body photons from the Keplerian disk, with the power-law component from thermal Comptonization, one obtains a complete spectrum. The Comptonized photons then follow curved trajectories as they leak out from the CENBOL after possible repeated scatterings and reach the observer. Higher energy X-rays are expected to come from deep inside of the CENBOL after multiple scatterings. Thus, one can obtain a different image with photons of different energy range. This makes the problem very interesting as far as observations are concerned. From the same simulation, by filtering photons reaching out to a series of observers sitting at different inclination angles, we also obtain totally different images and spectra of the same source with the same intrinsic disk properties. The time lag properties of this configuration would be important also. This would be reported elsewhere.

The plan of our paper is the following. In the next Section, we discuss geometry of the CENBOL and the Keplerian disk in our simulation. In $\S 3$, we describe the equations governing the null trajectories and image generation process that were used in this work. In $\S 4$, we present results of Monte-Carlo simulations for different disk parameters and in $\S 5$, we draw conclusions.

\section{NATURE OF THE COMPTON CLOUD AND THE SOFT PHOTON SOURCE}

In TCAF scenario, the higher viscosity component forms a Keplerian disk while the lower viscosity and low angular momentum component (which surrounds the Keplerian flow) forms a centrifugal barrier close to a black hole. In a large region of the parameter space, the centrifugal barrier induces formation of a standing or an oscillatory shock. The post-shock region is the Compton cloud. Being subsonic and basically rotating with more or less constant angular momentum, CENBOL satisfies some of the conditions of a radiation pressure supported thick accretion disk or a hot ion pressure supported torus (Abramowicz et al., 1978; Kozlowski et al., 1978; Paczyński \& Wiita, 1980; Begelman, Blandford \& Rees, 1982; Chakrabarti, 1985, hereafter C85) and therefore for all practical purposes, one can use C85 prescription of 'natural angular momentum' in general to describe electron number density and temperature distribution inside a hot CENBOL. CENBOL remains a hot ion pressure 


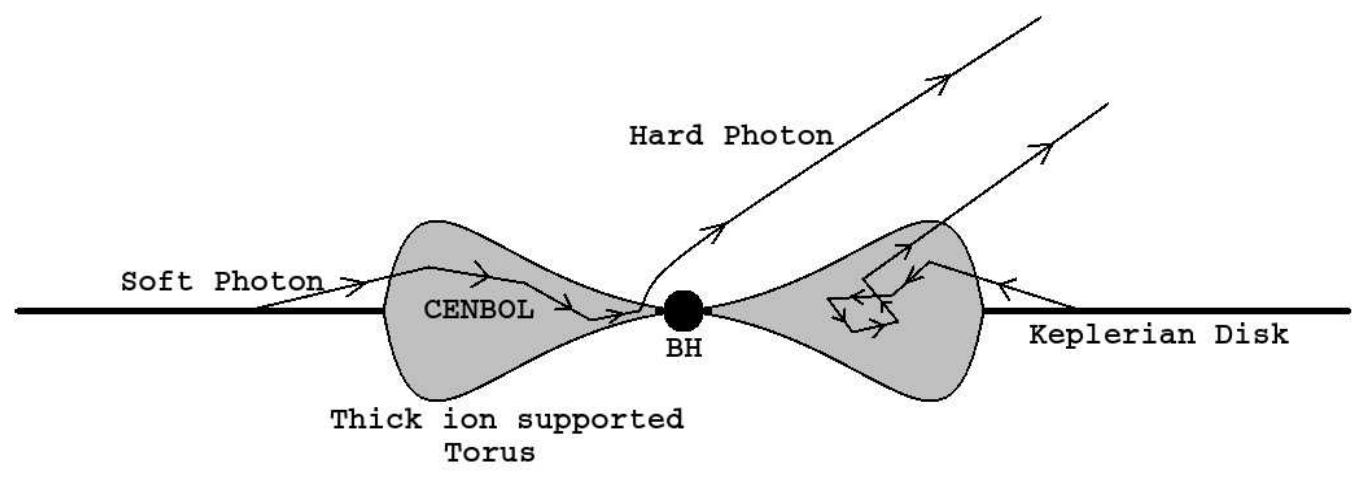

Figure 1. Our simulation geometry of two component advective flow solution consisting of one high viscosity truncated Keplerian disk component (Page \& Thorne 1974) and one low angular momentum Polish donut type thick ion supported torus component (C85). Typical photon trajectories of soft and hard photons are shown. The advective component before the shock outside CENBOL is tenuous and is ignored.

supported torus when the Keplerian component has a little accretion rate and the number of soft photon is not enough to cool down the CENBOL. We follow the procedure given in C85 to obtain the temperature and density distribution inside the CENBOL and treat it as the Compton cloud. In TCAF, the outer edge of the CENBOL is also the inner edge of the Keplerian component. Soft photons from the Keplerian disk is intercepted by the CENBOL and are re-emitted after Comptonization. In very soft states, when the Keplerian component rate is high, the CENBOL collapses and the entire disk can be treated as the standard Shakura-Sunyaev (1973, hereafter SS73) type disk which will pass through the inner sonic point due to its nature of transonicity (Chakrabarti, 1990). With even higher accretion rates, the inner edge is puffed up to produce a radiation pressure supported torus, but we will not explore that regime here. In Fig. 1, we present the geometry of our simulation. In order to obtain a stationary solution, we replace the high viscosity component of TCAF by the standard Keplerian disk as suggested by Page \& Thorne (1974) (relativistic counterpart of SS73) and the advecting CENBOL by a purely rotating thick ion torus (C85). Typical schematic photon trajectories are shown.

\subsection{CENBOL as a relativistic torus}

To describe CENBOL, we note that the relativistic Euler's equation for the perfect fluid under the influence of gravity, centrifugal force and pressure gradient force can be written as $(\mathrm{C} 85)$,

$$
\frac{\nabla p}{p+\epsilon}=-\ln \left(u_{t}\right)+\frac{\Omega \nabla l}{(1-\Omega l)}
$$


where, $p$ is the pressure and $\epsilon$ is the total energy density. Also, $l=-u_{\phi} / u_{t}$ is specific angular momentum and $\Omega=u^{\phi} / u^{t}$ is the relativistic angular momentum. Velocity components $u^{r}$ and $u^{\theta}$ are negligible for the present scenario. We consider barotropic process $p=p(\epsilon)$ such that constant pressure surfaces and equipotential surfaces coincides. Then, Euler's equation becomes,

$$
W-W_{i n}=\int_{0}^{p} \frac{d p}{p+\epsilon}=\int_{u_{t i n}}^{u_{t}} \ln \left(u_{t}\right)-\int_{l_{i n}}^{l} \frac{\Omega \nabla l}{(1-\Omega l)},
$$

where, for a given angular momentum distribution $\Omega=\Omega(l)$, we can easily integrate the third term of this equation.

The ratio $l / \Omega=\lambda^{2}$ has dimension $\left[L^{2}\right]$. Choosing $l=c \lambda^{n}(\mathrm{C} 85)$, where $c$ and $n$ are constants, yields the von-Zeipel relationship as follows,

$$
\Omega=c^{2 / n} l^{1-2 / n},
$$

and $\lambda=\frac{r \sin \theta}{\left(1-\frac{2}{r}\right)^{1 / 2}}(\mathrm{C} 85)$. In our simulation, we choose $G=1, M=1$ and $c=1$ such that $r_{g}=2$.

Constant parameters $c$ and $n$ can be derived from the following conditions (C85):

$$
\begin{array}{r}
c \lambda_{i n}^{n}=l_{k}\left(r=r_{i n}, \theta=\pi / 2\right) \text { and } \\
c \lambda_{c}^{n}=l_{k}\left(r=r_{c}, \theta=\pi / 2\right),
\end{array}
$$

where, the subscript in and $c$ correspond to the quantities at the disk inner edge and disk center. We kept $r_{i n}=4.0$ and $r_{c}=9.8$ to generate the innermost thick toroidal shell. Variation of the potential keeping $r_{i n}$ and $r_{c}$ fixed gives a large number of shells which create the complete stationary CENBOL. Comptonization occurs inside this region. Since, electrons inside the disk generally have higher energy than the soft photons injected into it, the later are repeatedly inverse Comptonized before they leave the CENBOL and reach the observer in curved trajectories.

\subsection{Distribution of temperature and density inside the Compton cloud}

For Comptonization, we need the information of density, pressure and temperature inside the CENBOL region. For this, we choose a barotropic equation of state where iso-temperature and iso-density surfaces coincide with equipotential surfaces. We calculate the disk temperature using a polytropic equation of state, $p=K \rho^{\gamma}$ where $p, \rho, K$ and $\gamma$ are pressure, matter density a constant related to entropy and polytropic index respectively. In a relativistic flow, one can choose $\gamma=4 / 3$. Since we are interested to study the effects of gravitational bending, we fix the CENBOL properties such that at $r=r_{c}$, the temperature is $\sim 200 \mathrm{keV}$ and the 

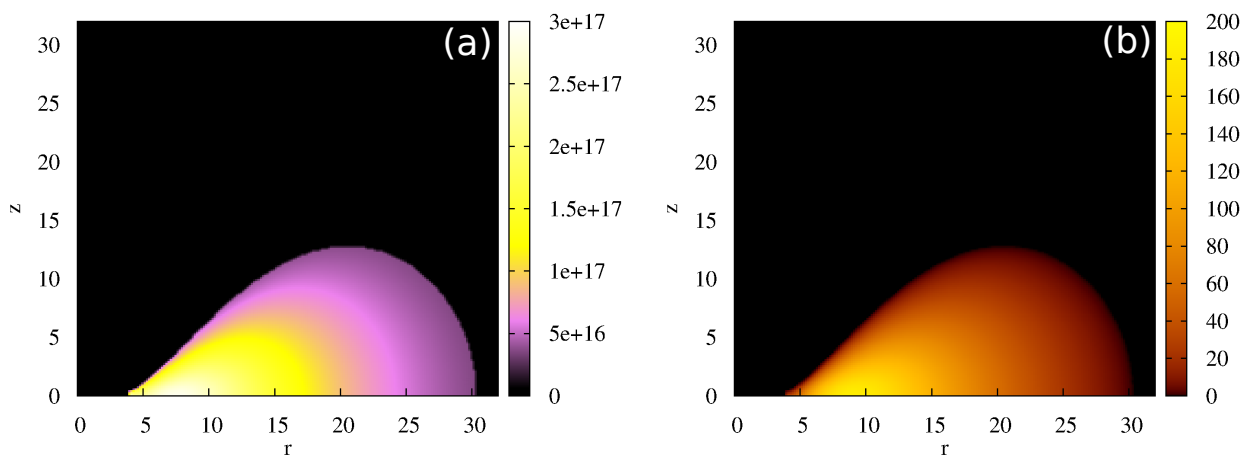

Figure 2. (a) Number density and (b) temperature profile of the CENBOL with inner edge at 4.0, center at 9.8 and outer edge at 30. Temperature is given in keV unit. Pressure, number density \& temperature of the outermost layer is kept at zero.

highest electron number density is $\sim 3.0 \times 10^{17}$. These can be achieved using a suitable entropy constant, $K$ as $T \propto \rho^{1 / 3}$. In Figure 2 we have shown the variation of electron number density (per $\mathrm{cm}^{3}$ ) and temperature (in $\mathrm{keV}$ ) inside the CENBOL.

\subsection{Soft Photon Source}

Keplerian disk acts as the source of soft photons. We consider the disk outer edge to be only up to 50 so as to save computing time. The inner edge is truncated just outside the CENBOL boundary. We assume this disk to be sitting on the equatorial plane. We use the simplified radiation profile as a function of radius as given by Page \& Thorne (1974). Bolometric flux of radiation from a Keplerian disk around a Schwarzschild black hole is written as:

$$
F_{k}^{d i s k}(r)=\frac{F_{c}\left(\dot{m}_{d}\right)}{(r-3) r^{5 / 2}}\left[\sqrt{r}-\sqrt{6}+\frac{\sqrt{3}}{3} \log \left(\frac{(\sqrt{r}+\sqrt{3})(\sqrt{6}-\sqrt{3})}{(\sqrt{r}-\sqrt{3})(\sqrt{6}+\sqrt{3})}\right)\right],
$$

where, $F_{c}\left(\dot{m}_{d}\right)=\frac{3 m \dot{m}_{d}}{8 \pi r_{g}^{3}}$, with $\dot{m}_{d}$ being the disk accretion rate in Eddington unit. From Stefan-Boltzmann law, one can easily get local temperature of the disk by,

$$
T_{k}^{d i s k}(r)=\left(\frac{F_{k}^{d i s k}(r)}{\sigma}\right)^{1 / 4}
$$

where, $\sigma=\frac{2 \pi^{5} k^{4}}{15 h^{3} c^{3}}$ is the Stefan-Boltzmann constant. Total number of photons emitted from Keplerian disk surface is given by

$$
n_{\gamma}(r)=\left[16 \pi\left(\frac{k_{b}}{h c}\right)^{3} \times 1.202057\right]\left(T_{k}^{d i s k}(r)\right)^{3}
$$

The number of photons emitted from the radius $r$ to $r+\delta r$ is given by

$$
d N(r)=4 \pi r \delta r H(r) n_{\gamma}(r)
$$

where Keplerian disk height $H(r)$ is assumed to be 0.1 (as $\frac{H(r)}{r}<<1$ for Keplerian disk region). The Keplerian disk is divided in different annuli of width $D(r)=0.1$. Each annulus having mean radius $\mathrm{r}$ is characterized by its average temperature $T_{k}^{d i s k}(r)$. The total number 
of photons emitted from the disk surface of each annulus can be calculated using Equation (8). This total number comes out to be $\sim 10^{39}-10^{40}$ per second for $\dot{m}_{d}=0.1$. One cannot inject this many photons in Monte Carlo simulation because of the limitation of computation time. So we replace this large number of photons by a lower number of bundles of photons, $N_{\text {comp }}(r)=10^{9}$, and calculate a weightage factor $f_{W}=\frac{d N(r)}{N_{c o m p}(r)}$.

For different annulus, the number of photons in a bundle will change. From the standard disk model of Page and Throne (1974), we calculate $d N(r)$ and use that to calculate the change in energy via Comptonization. During inverse-Comptonization (or, Comptonization) by an electron in an elemental volume of $d V$, we consider that $f_{W}$ number of photons has suffered the same scattering with the electrons inside the same volume (Garain, Ghosh \& Chakrabarti 2012).

Monte-Carlo code randomly generates soft photons from the Keplerian disk. Soft photon energy is calculated using Planck distribution law for a given $T_{k}^{\text {disk }}(r)$. The number density of photons $\left(n_{\gamma}(E)\right)$ having an energy $E$ is given by,

$$
n_{\gamma}(E)=\frac{1}{2 \zeta(3)} b^{3} E^{2}\left(e^{b E}-1\right)^{-1},
$$

where, $b=1 / k T_{k}^{d i s k}(r)$ and $\zeta(3)=\sum_{1}^{\infty} l^{-3}=1.202$, the Riemann zeta function (e.g., Ghosh, Chakrabarti \& Laurent, 2009).

\subsection{Simulation Procedure}

To reduce the Computational time we consider the trajectories of photons to be straight lines inside the CENBOL. Difference of results with null geodesic path in between scatterings and straight line path was shown to be insignificant (Laurent \& Titarchuk, 1999). The injected soft photons are generated from the Keplerian disk at a random direction which required use of three random numbers. In the Monte Carlo simulation, the intensity of emitted radiation from the Keplerian disk $\propto \cos \theta$. That implies the Keplerian disk will radiate most along the Z-direction and least along the equatorial plane.

Another random number gives a target optical depth $\tau_{c}$ at which the scattering occurs. The photon is tracked within the electron cloud till the optical depth $(\tau)$ reached $\tau_{c}$. The probability density of Compton scattering of a photon with an electron $\propto e^{-\tau}$, where $\tau$ is the optical depth of the cloud at that particular position. We calculate $\tau_{c}$ by randomizing this particular function using Monte Carlo technique. Finally, we get $\tau_{c}=-\ln (\zeta)$, where $\zeta$ is a uniform random number between 0 and 1 . The increase in optical depth $(d \tau)$ as the 
photon travels a path of length $d l$ inside the electron cloud is given by: $d \tau=\rho_{n} \sigma d l$, where $\rho_{n}$ is the local electron number density.

The total scattering cross section $\sigma$ is given by Klein-Nishina formula:

$$
\sigma=\frac{2 \pi r_{e}^{2}}{x}\left[\left(1-\frac{4}{x}-\frac{8}{x^{2}}\right) \ln (1+x)+\frac{1}{2}+\frac{8}{x}-\frac{1}{2(1+x)^{2}}\right]
$$

where, $x$ is given by,

$$
x=\frac{2 E}{m c^{2}} \gamma\left(1-\mu \frac{v}{c}\right)
$$

$r_{e}=e^{2} / m c^{2}$ is the classical electron radius and $m$ is the mass of the electron.

We assumed here that a photon of energy $E$ and momentum $\frac{E}{c} \widehat{\Omega}$ is scattered by an electron of energy $\gamma m c^{2}$ and momentum $\overrightarrow{\mathbf{p}}=\gamma m \overrightarrow{\mathbf{v}}$, with $\gamma=\left(1-\frac{v^{2}}{c^{2}}\right)^{-1 / 2}$ and $\mu=\widehat{\mathbf{\Omega}} \cdot \widehat{\mathbf{v}}$. At this point, a scattering is allowed to take place. The photon selects an electron and the energy exchange is computed using the Compton or inverse Compton scattering formula. The electrons are assumed to obey relativistic Maxwell distribution inside the Compton cloud. The number $d N(p)$ of Maxwellian electrons having momentum between $\vec{p}$ to $\vec{p}+d \vec{p}$ is expressed by,

$$
d N(\vec{p}) \propto \exp \left[-\left(p^{2} c^{2}+m^{2} c^{4}\right)^{1 / 2} / k T_{e}\right] d \vec{p}
$$

While travelling from one scattering centre to another, the gravitational redshift changes the frequency of the photon. The process continues until the photon leaves CENBOL region or is sucked in by the black hole. The process is similar to what was used in Ghosh, Chakrabarti \& Laurent (2009), Ghosh et al. (2010, 2011), Garain et al. (2012), (2014); which was adopted originally from Pozdnyakov, Sobol and Sunyaev (1983). We choose the mass of the black hole $\left(M=10 M_{\odot}\right)$ in our simulation. In Fig. 3a, the energy and in Fig. 3b, number of scattering suffered by an emergent photon is shown. The higher energy photons are generated after relatively higher number of scatterings. The highest possible temperature is located at the centre of the disk $r_{c}$. The smooth energy contours like Fig. 3a can only be found for inclination independent cross-sectional view of the CENBOL. To an observer at a particular inclination angle, only a fraction of those photons reach the eye. Thus we get a rather noisier image. 

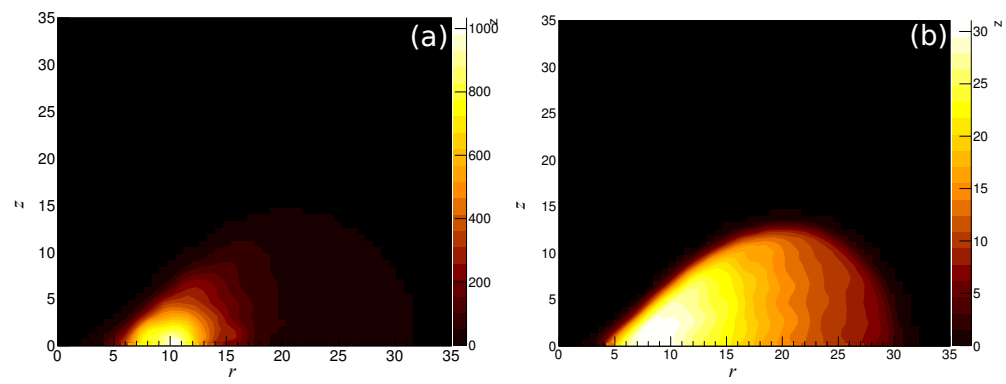

Figure 3. (a) Energy and (b) number of scattering suffered by emergent photons from the CENBOL. Photons which escape from the centre of CENBOL suffered maximum number of scattering. They generally reach to highest energies due to Comptonization.

\section{SPACE-TIME METRICS USED AND THE IMAGE GENERATION PROCESS}

In this Section, we describe our method of image generation process in details. For simplicity of the problem we choose the Schwarzschild geometry. This describes the spacetime around a non-rotating, uncharged, spherically symmetric object.

The metric components are assigned as (Weinberg, 1972), $g_{t t}=f(r)=\left(1-\frac{2}{r}\right), g_{r r}=$ $h(r)=f(r)^{-1}, g_{\theta \theta}=-r^{2}, g_{\phi \phi}=-r^{2} \sin ^{2} \theta$ and the inverse metric components as $g^{t t}=$

$-f(r)^{-1}, g^{r r}=h(r)^{-1}, g^{\theta \theta}=r^{-2}, g^{\phi \phi}=r^{-2} \sin ^{-2} \theta$. Relation between curvature tensor and the Christoffel symbol follows

$$
\Gamma_{\nu \lambda}^{\mu}=\frac{1}{2} g^{\mu \xi}\left[\frac{\partial g_{\xi \nu}}{\partial x^{\lambda}}+\frac{\partial g_{\xi \lambda}}{\partial x^{\nu}}-\frac{\partial g_{\nu \lambda}}{\partial x^{\xi}}\right],
$$

where $\Gamma_{\nu \lambda}^{\mu}$ is the Christoffel symbols. Non vanishing components of Christoffel symbols are $\Gamma_{r r}^{r}=\frac{1}{2 h(r)} \frac{d h(r)}{d r}, \Gamma_{\theta \theta}^{r}=\frac{r}{h(r)}, \Gamma_{\phi \phi}^{r}=\frac{r^{2} \sin ^{2} \theta}{h(r)}, \Gamma_{r \theta}^{\theta}=\Gamma_{\theta r}^{\theta}=\Gamma_{r \phi}^{\phi}=\Gamma_{\phi r}^{\phi}=\frac{1}{r}, \Gamma_{\phi \phi}^{\theta}=-\sin \theta \cos \theta$, $\Gamma_{\theta \phi}^{\phi}=\Gamma_{\phi \theta}^{\phi}=\cot \theta$ and $\Gamma_{r t}^{t}=\Gamma_{t r}^{t}=\frac{1}{2 f(r)} \frac{d f(r)}{d r}$ similar to Weinberg (1972).

\subsection{Photon Trajectory Equations}

In four dimensions, the motion of free particles or photons are governed by the following equation,

$$
\frac{d^{2} x^{\mu}}{d p^{2}}+\Gamma_{\nu \lambda}^{\mu} \frac{d x^{\nu}}{d p} \frac{d x^{\lambda}}{d p}=0
$$

with $\mu=[0,1,2,3] ; x^{0}=t, x^{1}=r, x^{2}=\theta$ and $x^{3}=\phi$, where $p$ is our affine parameter.

From Eqs (1), (2) and (3) we obtain four coupled, second order differential equations. 
They are as follows:

$$
\begin{aligned}
\frac{d^{2} t}{d p^{2}}+\frac{f^{\prime}(r)}{f(r)}\left(\frac{d t}{d p}\right)\left(\frac{d r}{d p}\right) & =0 \\
\frac{d^{2} r}{d p^{2}}+\frac{h^{\prime}(r)}{2 h(r)}\left(\frac{d r}{d p}\right)^{2}-\frac{r}{h(r)}\left(\frac{d \theta}{d p}\right)^{2}-\frac{r \sin ^{2} \theta}{h(r)}\left(\frac{d \phi}{d p}\right)^{2}+\frac{f^{\prime}(r)}{2 h(r)}\left(\frac{d t}{d p}\right)^{2} & =0 \\
\frac{d^{2} \theta}{d p^{2}}+\frac{2}{r}\left(\frac{d \theta}{d p}\right)\left(\frac{d r}{d p}\right)-\sin \theta \cos \theta\left(\frac{d \phi}{d p}\right)^{2}=0 & \text { and } \\
\frac{d^{2} \phi}{d p^{2}}+\frac{2}{r}\left(\frac{d \phi}{d p}\right)\left(\frac{d r}{d p}\right)+2 \cot \theta\left(\frac{d \theta}{d p}\right)\left(\frac{d \phi}{d p}\right) & =0
\end{aligned}
$$

We introduce energy $P_{t}=E=\left(1-\frac{2}{r}\right) \frac{d t}{d p}$ and angular momentum $P_{\phi}=L=r^{2} \sin ^{2} \theta \frac{d \phi}{d p}$ which are two physical quantities for each photon (Chandrasekhar, 1983). Since the path of photons are independent of energy, we consider $P_{t}=E=1$. On the other hand, $P_{\phi}=L$ is a variable which essentially produces photons of various impact parameters as defined by $b=L / E$ (Luminet 1979).

Without the lose of generality, we can drop one equation by substituting the derivative from affine parameter $p$ to time $t$ co-ordinate. Thus we get

$$
\begin{array}{r}
\frac{d^{2} r}{d t^{2}}+\frac{3}{r(r-2)}\left(\frac{d r}{d t}\right)^{2}-(r-2)\left(\frac{d \theta}{d t}\right)^{2}-(r-2) r \sin ^{2} \theta\left(\frac{d \phi}{d t}\right)^{2}+\frac{r-2}{r^{3}}=0 \\
\frac{d^{2} \theta}{d t^{2}}+\frac{2 r-6}{r(r-2)}\left(\frac{d \theta}{d t}\right)\left(\frac{d r}{d t}\right)-\sin \theta \cos \theta\left(\frac{d \phi}{d t}\right)^{2}=0 \text { and } \\
\frac{d^{2} \phi}{d t^{2}}+\frac{2 r-6}{r(r-2)}\left(\frac{d \theta}{d t}\right)\left(\frac{d r}{d t}\right)+2 \cot \theta\left(\frac{d \theta}{d t}\right)\left(\frac{d \phi}{d t}\right)=0
\end{array}
$$

Finally, we have three second order coupled differential equations which governs the path of a photon in this curved geometry.

\subsection{Velocity Components: Tetrad formalism}

The formalism of Tetrad is used to connect various coordinates. The nature of Tetrad frame is locally inertial (Park, 2006). By virtue of this, it connects physical quantities in curved spacetime to a flat spacetime. Fixed and co-moving Tetrad are of most importance. However, here, we shall concentrate our studies for fixed Tetrad. Let us take an orthonormal fixed Tetrad (for our observer) with basis vector $e_{\hat{\mu}}=\partial / \partial \hat{x}^{\mu}$. The connection to another coordinate 
with unit vector $e_{\mu}=\partial / \partial x^{\mu}$ is established via following relations (Weinberg 1972):

$$
\begin{aligned}
\frac{\partial}{\partial \hat{t}} & =\frac{1}{f(r)^{1 / 2}} \frac{\partial}{\partial t} \\
\frac{\partial}{\partial \hat{r}} & =f(r)^{1 / 2} \frac{\partial}{\partial r} \\
\frac{\partial}{\partial \hat{\theta}} & =\frac{1}{r} \frac{\partial}{\partial \theta} \text { and } \\
\frac{\partial}{\partial \hat{\phi}} & =\frac{1}{r \sin \theta} \frac{\partial}{\partial \phi} .
\end{aligned}
$$

These transformations allow us to connect physical quantities from the curved to the flat spacetime. From above equations one can deduce three spatial velocity components which can be expressed as,

$$
\begin{array}{r}
v^{\hat{r}}=\frac{d \hat{r}}{d t}=\frac{r}{(r-2)} \frac{d r}{d t}, v^{\hat{\theta}}=\frac{d \hat{\theta}}{d t}=\frac{r \sqrt{r}}{\sqrt{(r-2)}} \frac{d \theta}{d t} \\
\text { and } v^{\hat{\phi}}=\frac{d \hat{\phi}}{d t}=\frac{r \sqrt{r} \sin \theta}{\sqrt{(r-2)}} \frac{d \theta}{d t} .
\end{array}
$$

\subsection{Solving Geodesic Equations}

We solve these three differential equations using fourth order Runge - Kutta method where initial velocity components are supplied in Cartesian co-ordinate. The unitary transformation matrix between $\left(v^{\hat{x}}, v^{\hat{y}}, v^{\hat{z}}\right)$ and $\left(v^{\hat{r}}, v^{\hat{\theta}}, v^{\hat{\phi}}\right)$ is given below,

$$
\left(\begin{array}{c}
v^{\hat{r}} \\
v^{\hat{\theta}} \\
v^{\hat{\phi}}
\end{array}\right)=\left(\begin{array}{ccc}
\sin \theta \cos \phi & \sin \theta \sin \phi & \cos \theta \\
\cos \theta \cos \phi & \cos \theta \sin \phi & -\sin \theta \\
-\sin \theta & \cos \phi & 0
\end{array}\right)\left(\begin{array}{c}
v^{\hat{x}} \\
v^{\hat{y}} \\
v^{\hat{z}}
\end{array}\right)
$$

The initial positions $\left(x_{\circ}^{\nu}\right.$, where $\left.\nu=1,2,3\right)$ and velocities $\left(\left[\frac{d \hat{x}^{\nu}}{d t}\right]_{0}\right.$, with $\left.\nu=1,2,3\right)$ of emergent photons are supplied from the Monte-Carlo Comptonization code. To obtain images for a particular inclination angle, we combine all the photons obtained from various azimuthal angles to increase the intensity of the image. The rotational symmetry in Schwarzschild geometry allows us to do this superposition without a loss of generality.

\subsection{Redshifts of Photons}

To generate spectrum and the image one must introduce the redshift. In this scenario, there are two parts of the redshift. One part comes from the Doppler effect caused by rotational motion of the disk matter and the other part is the gravitational redshift caused by the 


\section{Arka Chatterjee, Sandip K. Chakrabarti \& Himadri Ghosh}

black hole. So, the net redshift can be written as,

$$
1+z=r_{\text {grav }} r_{\text {rot }}
$$

where, $r_{\text {grav }}$ and $r_{\text {rot }}$ are gravitational and rotational contributions to redshift respectively. As a whole, one can express redshift as,

$$
1+z=\frac{E_{e m}}{E_{o b s}}=\frac{\left(P_{\alpha} u^{\alpha}\right)^{e m}}{\left(P_{\alpha} u^{\alpha}\right)^{o b s}}
$$

where, $E_{e m}$ and $E_{o b s}$ are the energy of emitted and observed photons respectively. Now, taking an inner product of 4-momentum and 4-velocity gives the energy of emitted photon as

$$
E_{e m}=P_{t} u^{t}+P_{\phi} u^{\phi}=P_{t} u^{t}\left(1+\Omega_{\phi} \frac{P_{\phi}}{P_{t}}\right),
$$

where $\Omega_{\phi}=\frac{u^{\phi}}{u^{t}}$ (for Keplerian disk $\Omega_{\phi}=\left(1 / r^{3}\right)^{1 / 2}$ ) and for CENBOL region $\Omega_{\phi}=c \lambda^{n-2}$ ). From earlier definition $\frac{P_{\phi}}{P_{t}}=\frac{L}{E}$ and known as the projection of impact parameter along the z-axis. As in Luminet (1979), we consider the final form of redshift as,

$$
1+z=(1-3 / r)^{-1 / 2}\left(1+\Omega_{\phi} b \sin \theta_{\circ} \sin \alpha\right)
$$

where, $(1-3 / r)^{-1 / 2}=r_{\text {grav }}$ and $\left(1+\Omega_{\phi} b \sin \theta_{\circ} \sin \alpha\right)=r_{\text {rot }}$. The relation between the observed flux and the emitted flux is given by,

$$
F_{k}^{o b s}=\frac{F_{k}^{\text {disk }}}{(1+z)^{4}}
$$

Fourth power in the redshift factor comes due to energy loss of photons, gravitational time dilation and relativistic correction of detector solid angle. Similarly, the observed temperature will differ from the source temperature by,

$$
T_{k}^{o b s}=\left(\frac{F_{k}^{o b s}}{\sigma}\right)^{1 / 4}
$$

For a Keplerian disk, the maximum observed flux is located at $r\left(F_{k}^{\max }\right)=9.77$ with $F_{k}^{\max } \sim$ $1.763 \times 10^{-4} F_{c}\left(\dot{m}_{d}\right)$ (all accretion rates are measured in units of $\dot{m}_{d}=\frac{\dot{M}}{\dot{M}_{e d d}}$ ).

The Doppler effect increases as we increase the inclination angle. In case of $0^{\circ}$ inclination, there will not be any line splitting due to Doppler effect (Fabian et al., 2000). We show Doppler tomography for $0.01^{\circ}$ (Fig. 4a) inclination angle where the effect of differential rotation of the accretion disk is negligible and the observed spectra remain similar to the emitted spectra. In Fig. 4b, we see the effects of rotation for $82^{\circ}$ inclination, where the isoradial curves are deformed and observed spectra differ from what the disk is emitting. We consider all the photons in generating tomographic Figures. The image of the Keplerian disk is shown in Fig. 4c for an inclination angle of $82^{\circ}$. The color-bar represents a normalized 

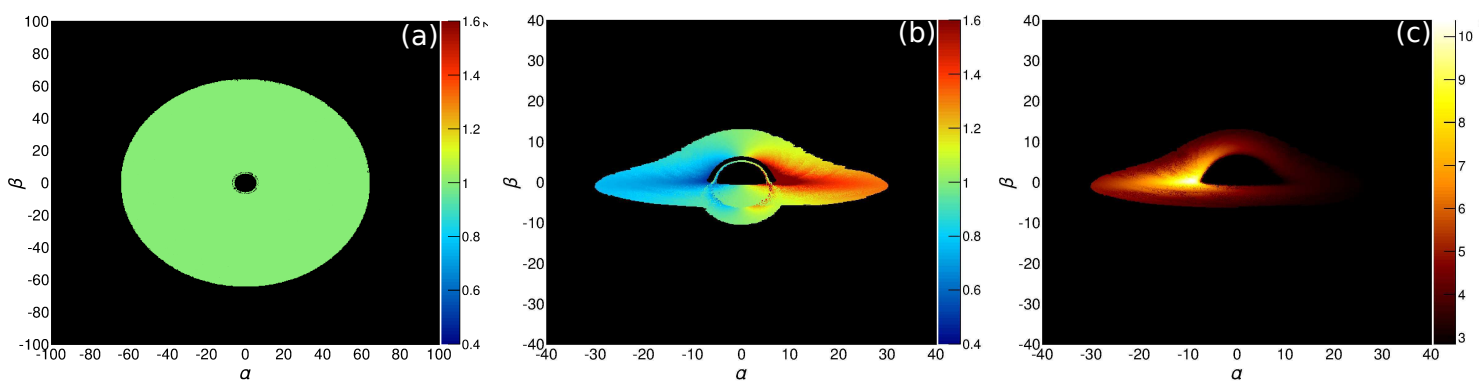

Figure 4. Doppler tomography of Keplerian disk having inclination angles (a) $0.01^{\circ}$ and (b) $82^{\circ}$ with outer edge $\left(r_{\text {out }}\right)$ at 60.0 and 30.0, respectively. Exact pole on view will give a complete zero Doppler effect disk. Colors represent contours of $\left(1+r_{\text {rot }}\right)$. Image of Keplerian disk with $r_{\text {out }}=30.0$ at an inclination angle $82^{\circ}$ is shown in (c). The color bar represents normalized temperature of the Keplerian disk. The inner edge $\left(r_{i n}\right)$ of the Keplerian disk is at 6.0 in all these three Figures.

observed temperature of Keplerian disk with the inner edge at 6.0 and outer edge at 30.0. We consider first order photons (i.e., photons which reach us directly without a complete rotation around the black hole) to construct this image. Because gravitational redshifts of photons which arrive at the observer after orbiting the black hole at least once or more is high, the contribution of those photons in a realistic image will be very low.

A picture similar to Fig. 4c has previously been presented in Fukue \& Yokoyama (1988) using the semi-analytical transfer function. The null trajectory solution (Eqn. 11) which we use, gives us the freedom to use three dimensional geometry and ability to gather information of physical quantities of that photon at any point in their path. The time of arrival of photons to an observer can be evaluated by this process. Using Monte Carlo technique, we produce an image, more realistic than the earlier images using semi-analytical transfer function methods.

\section{RESULTS AND DISCUSSIONS}

\subsection{Spectra}

Now that we have demonstrated our ability to image a standard disk, we now carry out the Monte-Carlo simulation including Comptonization and consider a TCAF configuration with a CENBOL and a truncated Keplerian disk as described in $\S 2$. We keep our detectors at 50 , just outside the TCAF system to get the simulated source spectrum, i.e., the spectrum that is just coming out of the source. For the generation of the image, the observer is placed at 100. In Fig. 5(a), the hardening of the output spectrum with the increase of the central temperature of the CENBOL is shown. Here, the disk rate $\left(\dot{m}_{d}\right)$ is kept fixed at 1.0 Eddington rate. For spectral study, We define the energy spectral index $\alpha$ to be $I(E) \propto E^{-\alpha}$. We note that $\alpha$ decreases, i.e., the spectrum hardens with the increase in the central temperature 

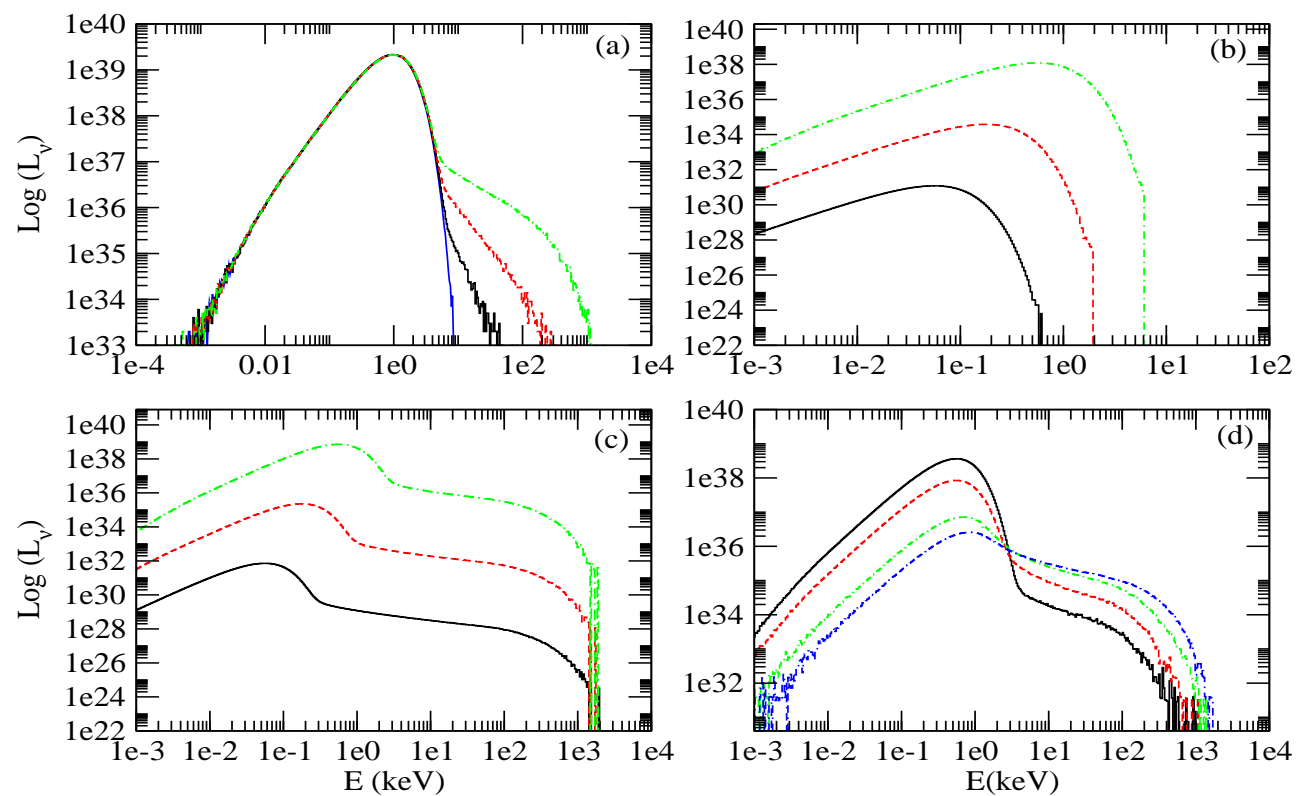

Figure 5. Variation of emergent spectrum with respect to (a) central temperature of CENBOL keeping $\dot{m}_{d}=1.0$ as fixed. The injected spectrum is shown by solid-blue line. The dashed-black, dot-dashed-red and double-dash-dot-green spectra correspond to the central temperatures 50, 100 and $140 \mathrm{keV}$, respectively. (b) Variation of injected seed photons from truncated disks of accretion rates $\dot{m}_{d}=0.00001$ (solid - black), $\dot{m}_{d}=0.001$ (dashed - red), $\quad \dot{m}_{d}=0.1$ (dot - dashed - green). The central temperature is $200 \mathrm{keV}$. (c) Emergent spectra with the seed photons as in (b). (d) Direction dependent emergent spectra for $\dot{m}_{d}=0.1$. The solid-black, dash-red, dot-dash-green and dot-dash-dash-blue curves represent inclination angles bins of $0^{\circ}-22.5^{\circ}, 22.5^{\circ}-45^{\circ}, 45^{\circ}-67.5^{\circ}$ and $67.5^{\circ}-90^{\circ}$ respectively.

Table 1. $\alpha$ variation with $T_{c} \& m_{d}$

\begin{tabular}{cccc}
\hline Figure & $\dot{m}_{d}$ & $T_{c}(\mathrm{keV})$ & $\alpha$ \\
\hline 5a (solid black) & 1.0 & 50.0 & 2.15 \\
5a (dashed red) & 1.0 & 100.0 & 1.81 \\
5a (dot dashed green) & 1.0 & 140.0 & 1.09 \\
\hline 5c (solid black) & 0.1 & 200.0 & 0.58 \\
5c (dashed red) & 0.001 & 200.0 & 0.55 \\
5c (dot dashed green) & 0.00001 & 200.0 & 0.54 \\
\hline
\end{tabular}

$\left(T_{c}\right)$ of CENBOL. The spectrum of the injected soft photons for accretion rates of $\dot{m}_{d}=$ 0.00001 (solid - black), $\dot{m}_{d}=0.001$ (dashed - red) and $\dot{m}_{d}=0.1$ (dot - dashed - green) are shown in Fig. 5b. The corresponding emergent spectra keeping the central temperature fixed at $200 \mathrm{keV}$ is shown in Fig. 5c. In Fig. 5d, the direction dependent source spectrum is shown. Photons are placed in four equal sized bins from 0 to 90 degrees $\left(0^{\circ}-22.5^{\circ}\right.$, $22.5^{\circ}-45^{\circ}, 45^{\circ}-67.5^{\circ}$ and $67.5^{\circ}-90^{\circ}$ respectively.) The spectrum is clearly getting harder for higher inclination angles. Variation of $\alpha$ with $T_{c}$ and $\dot{m}_{d}$ is presented also in Table 1. Also, $\alpha$ increases with increasing $\dot{m}_{d}$. The result is consistent with those of Chakrabarti \& Titarchuk (1995) carried out in the pseudo-Newtonian geometry. 


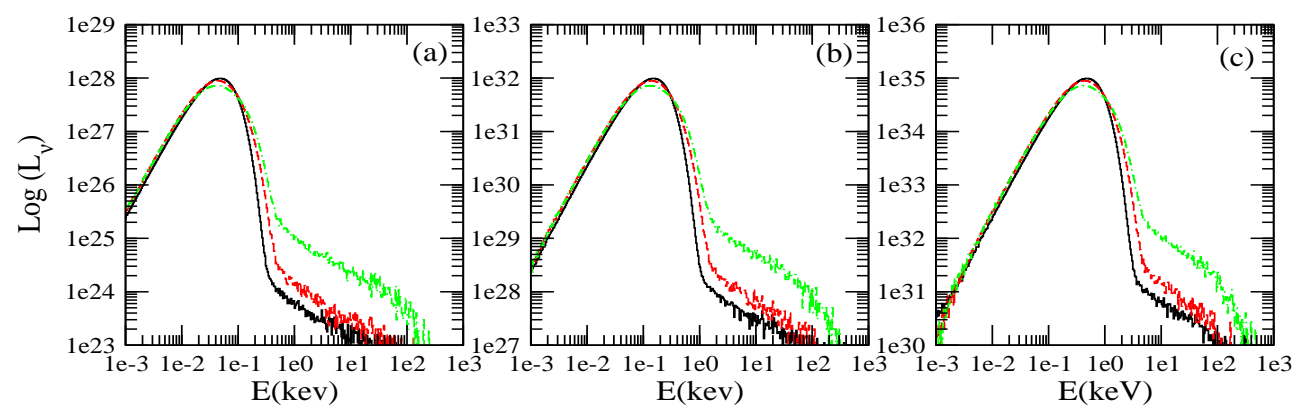

Figure 6. Variation of simulated observed spectrum with inclination angles $10^{\circ}$ (solid-black), $50^{\circ}$ (dot-red) and $80^{\circ}$ (dashgreen). Figs. (a), (b) and (c) corresponds to $m_{d}=10^{-5}, 10^{-3}$ and $10^{-1}$, respectively. Hardening of spectrum with the increase in inclination angle is clearly seen.

In Fig. 6, we show the variation of spectrum as seen by the observers sitting at three different inclination angles. The spectrum is getting harder as the inclination angle increases. This is clearly seen by the increase in the number of power-law photons for higher $\theta_{\text {obs }}$. For a photon to reach an observer at a higher $\theta_{o b s}$ it must pass through the CENBOL region and thus the probability of scattering increases. This makes the spectrum at higher $\theta_{\text {obs }}$ harder. We repeat the process for three different disk accretion rates and observe softening of the spectrum with the increase in $\dot{m}_{d}$.

Fig. 7 shows the variation of the ratio of $N_{p l}$ (the number of Comptonized photons) and $N_{b}$ (the number of black body photons) with inclination angles for accretion rates $\dot{m}_{d}=10^{-1}$ (solid black), $10^{-3}$ (dashed red) and $10^{-5}$ (dot-dashed-green) respectively. This ratio increases with decreasing accretion rate for a fixed inclination angle and increases with increasing inclination angle for a fixed accretion rate. The increment remains small for $10^{\circ}-50^{\circ}$ inclination. But, for $\theta_{o b s} \gtrsim 60^{\circ}$, the power-law photon number increases rapidly. The edge on view of CENBOL provides higher number of Comptonized photons. Thus, we can generally expect smaller spectral index or harder states and more spectral variabilities for higher inclination angle objects.

The black-body part of high inclination angle bin $\left(67.5^{\circ}-90^{\circ}\right)$ of the simulated source spectrum in Fig. 5d is much flatter than the low inclination angle case. But, in the simulated observed spectrum (Fig. 6) obtained at 100, the black-body part is much higher. Due to the shadowing effect of CENBOL, less number of black-body photons reach when we placed detectors just outside the system at 50 (Fig. 5d). The effect decreases with increasing observer distance. However, if the size of the CENBOL is large, the shadowing effect will also 


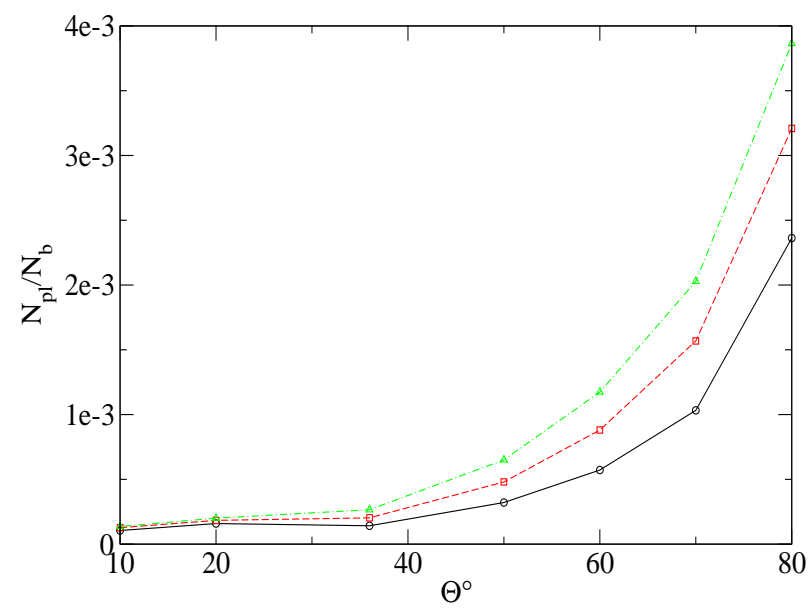

Figure 7. Variation of photon counts with inclination angle for accretion rates $m_{d}=10^{-1}$ (solid-black), $10^{-3}$ (dashed-red) and $10^{-5}$ (dot-dashed-green) is shown here. $N_{b}$ and $N_{p l}$ are black-body and power-law photon counts, respectively. As the inclination angle increases, the number of power-law photon increases. The black-body cut-offs for $m_{d}=10^{-1}, 10^{-3}$ and $10^{-5}$ accretion rates are considered as $3.5,1.0$ and $0.3 \mathrm{keV}$ respectively.
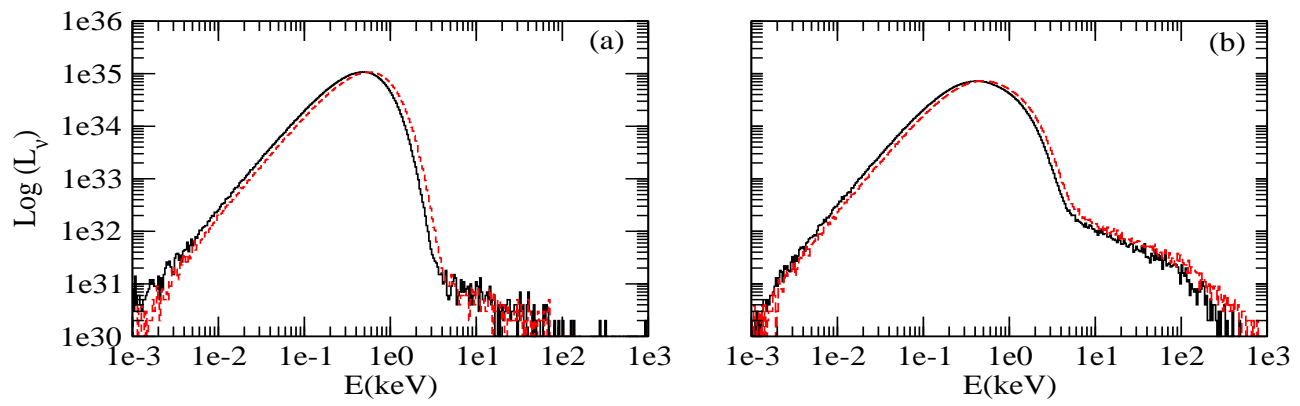

Figure 8. Variation of spectrum with and without gravitational bending for accretion rate $m_{d}=0.1$ is shown. In Fig. (a) and (b) inclination angles are $10^{\circ}$ and $80^{\circ}$, respectively. The solid-black line represents the spectra where gravitational bending is included. The dashed-red line represents where we considered straight line path for photons.

increase. This is a clear effect of photon bending where focussed photons which marginally escaped the CENBOL region arrive to observer at higher inclination angle.

We have shown simulated spectra as seen by an observer placed at $r=100$ for different accretion rates and inclination angles. We repeat the simulation by considering that the photons follow a straight line path as in a Newtonian space time; i.e., after excluding the effect of gravitational bending of light. We added Doppler effect because its outcome will still be present in the Newtonian regime. However, gravitational redshift is not included. We show the comparative spectra of two inclination angles having the same accretion rates $\dot{m}_{d}=0.1$ in Fig. 8. Due to the gravitational red-shift, the whole spectra for both cases 
shifted towards a lower energy range. Since the effect of gravitational redshift depends only on the mass of black hole, even after increasing inclination angle the difference between the two spectra remains the same.

\subsection{Images}

Image construction requires a one to one connection between an emitted photon at the source and an observed photon in the observer plane. This is established using geodesic relations. Photons scatter all around but the observer is generally localized $\left(r_{o b s}, \theta_{o b s}\right.$ and $\left.\phi_{o b s}\right)$. Exploiting the symmetry of the problem, we integrate over $\phi_{o b s}$ so as to merge observers with different $\phi_{o b s}$. Integration over elevation angle $\theta_{o b s}$ cannot be done. The distribution in $\theta_{\text {obs }}$ enable us to compare spectra and imaging at various inclination angles. A pole on view of the disk gives only the isoradial circles and the spectrum has no significant deviation from the source spectrum. The deviation of frequency and distortion in the isoradial curves became noticeable after $\theta_{\text {obs }}>30^{\circ}$ (Luminet, 1979).

The image of CENBOL with a Keplerian disk around is realistic as the spectra of the combined flow explains the observations most successfully. In Fig. 9, we present images of TCAF at various inclination angles. We used all the photons which appeared at a given inclination angle in constructing these images. Here, we demonstrate our results at three inclination angles with different accretion rates. As the accretion rate is increased, the disk temperature increases. The peak energy of black-body shifts towards higher value. The intensity also increases. So, the disk color becomes brighter emitting higher frequency radiation with accretion rates. We have considered the temperature range such that all the photons from the disk and CENBOL can be shown together in a single plot where temperature contours of disk are uniformly binned. The band structure on the Keplerian disk is due to this binning. With increasing disk rate, the minimum energy of Comptonized photon increases. These photons are mostly generated on the outer boundary of CENBOL. Color variation of the CENBOL region in Fig. 9 (from left to right accretion rate increases) is caused mostly due to the photons that are coming from the outer regions of the CENBOL. For high inclination $\left(\theta_{o b s}=80^{\circ}\right)$, Fig. 9g shows near disappearance of many Keplerian disk photons which were coming from the other side of the disk for a low disk accretion rate $\left(\dot{m}_{d}=0.00001\right)$. As the accretion rate goes down, the percentage of scattered photons increases. Since the photons from the opposite side (of the observer) which are coming towards the observer at 

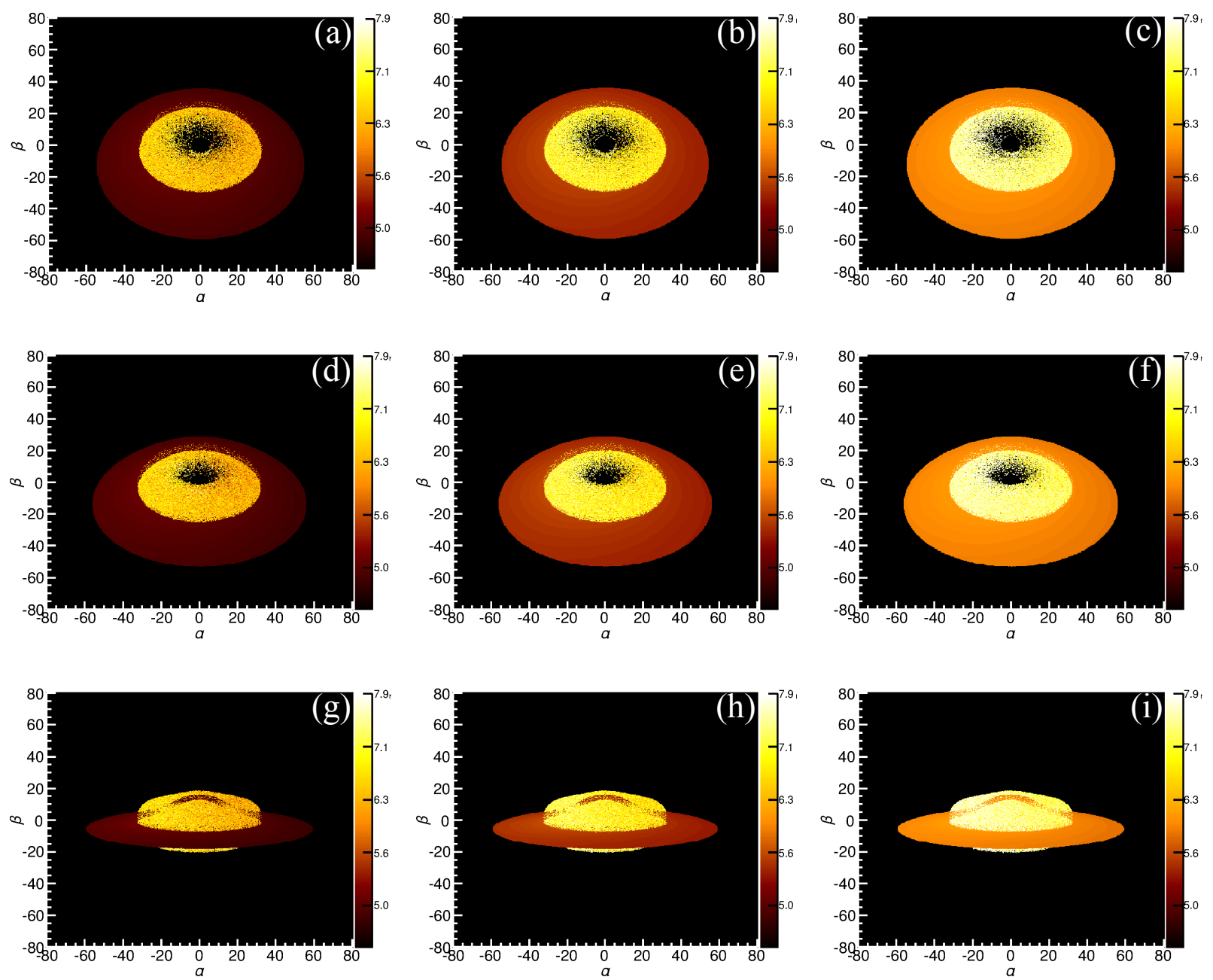

Figure 9. Images seen by observers from (top panel) $36^{\circ}, 50^{\circ}$ (middle panel), $80^{\circ}$ (bottom panel). Disk rates in the first, second and last column are $m_{d}=0.00001, m_{d}=0.001$ and $\dot{m}_{d}=0.1$ respectively. Color-bar is plotted in the $\log \left(T_{o b s}\right)$ scale with $10^{4.5}-10^{7.9}$ Kelvin is the range fixed for each Figure.

an higher inclination angle must pass through the CENBOL region, they are more scattered. This interesting effect due to focusing could also be tested observationally in future, for high inclination sources. Interestingly, with an increase in the disk rate, the number of photons passing through the CENBOL increases and the CENBOL appears to be splitted into two halves especially at high inclination angle. Note that the number of photons from the inner edge of the CENBOL is very few and they are either sucked into the black hole or are absorbed. Note that the inner edge of CENBOL is noisy especially for lower accretion rates, since very few photons are intercepted by the CENBOL in the first place and even fewer are emitted from this region. This noisy behavior is inherent to Monte-Carlo simulations and would go away with a larger sample of injected photons and also after smoothening out over finite detector pixels. The occasional color fluctuations of photons which appear to emerge 

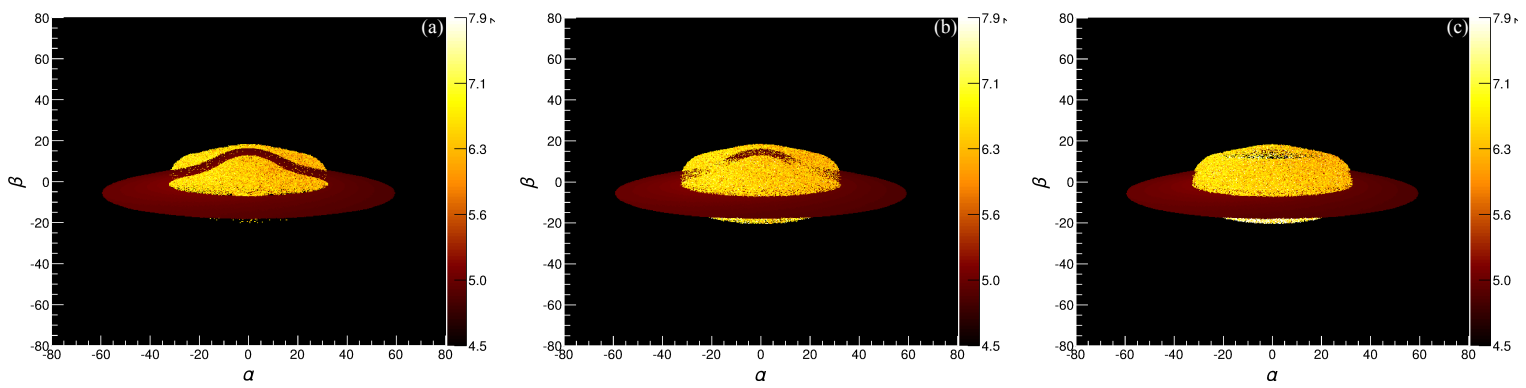

Figure 10. Images seen by observers from $80^{\circ}$. Disk rate is kept constant at $\dot{m}_{d}=0.00001$. The central temperature and density increases from left to right (Table 2): (a) $T_{c}=100 \mathrm{keV} ; n_{c}=4 \times 10^{16} \mathrm{~cm}^{-3} ;$ (b) $T_{c}=200 \mathrm{keV} ; n_{c}=3 \times 10^{17} \mathrm{~cm}^{-3} ;($ a) $T_{c}=300 \mathrm{keV} ; n_{c}=1.2 \times 10^{18} \mathrm{~cm}^{-3}$. Color-bar is plotted in the $\log \left(T_{\text {obs }}\right)$ scale with $10^{4.5}-10^{7.9}$ Kelvin is the range fixed for each case.

Table 2. Variation of images with $T_{c} \& n_{c}$

\begin{tabular}{cccc}
\hline Figure & $m_{d}$ & $T_{c}(\mathrm{keV})$ & $n_{c}\left(\right.$ per $\left.\mathrm{cm}^{3}\right)$ \\
\hline $10 \mathrm{a}$ & 0.000001 & 100.0 & $4 \mathrm{E} 16$ \\
$10 \mathrm{~b}$ & 0.000001 & 200.0 & $3 \mathrm{E} 17$ \\
$10 \mathrm{c}$ & 0.000001 & 300.0 & $1.2 \mathrm{E} 18$ \\
\hline
\end{tabular}

side by side are due to the fact that they are originated from two different optical depths which may have different temperatures, gravitational redshifts and energy transfer through Comptonization. This is to be contrasted with the smooth image of a spherical Comptonizing corona having a constant temperature and optical depth as obtained by Schnittman \& Krolik (2009).

There are other effects in our image which need some attention. The high energy photons (yellow) which are visible on the top of the distant half of the Keplerian disk in Fig. 9(a-f) are simply due to warped nature of the CENBOL. Just as in Fig. 4b where distant side of the Keplerian disk appeared to be warped, the distant side of CENBOL here also appears to be warped with the moderately warped Keplerian disk in the background. It is to be noted that the CENBOL emits only those photons intercepted from the Keplerian disk and are much fewer in number and thus the more intense, low temperature, Keplerian disk can still be seen peeping through the CENBOL, specially when the optical depth is low. For high inclination angle, only some of these Keplerian photons are scattering by relatively tenuous CENBOL and thus the warped Keplerian disk appears to be cutting the CENBOL into two parts. The front side of CENBOL is also cut into two halves mainly because the outer edge of the Keplerian disk is chosen to be at $50 G M / c^{2}$. A larger outer edge or a lower optical depth of the CENBOL would have removed the lower half of the CENBOL completely.

The optical depth of the CENBOL medium quantifies the number of scatterings that 
should occur. In Fig. 10 (a-c), we have shown three cases where densities and temperatures of electron cloud were self consistently varied inorder to study CENBOL with three different optical depths. The parameters employed are given in Table 2. In the lower density case, i.e., in Fig. 10a, photons from Keplerian disk have undergone very few scatterings in the CENBOL region. The optical depth of the medium is so low that almost all the photons from the far side of the Keplerian disk are visible by the observer. Since the number of scattering is small, very less number of CENBOL photons are visible from lower side of the Keplerian disk also. Fig. 10b drawn for an intermediate optical depth, is same as the Fig. 9g. Here some Keplerian photons cross over the electron cloud without scattering, especially in regions of lower optical depth. In Fig. 10c, we consider a relatively higher central density so that the optical depth is everywhere larger than unity. Here, almost all the photons which came from the other side of Keplerian disk participate in scattering and thus the Keplerian disk from far side becomes invisible.

Photons from the disk and the CENBOL thus participate in the formation of the photon sphere. Although the contribution of such photons is very less in the spectrum, the measurement of size and shape of the photon sphere can determine both the mass and spin of the black hole candidate in future. We will discuss this elsewhere.

\section{CONCLUSIONS}

Photons emerging from the vicinity a black hole encounter the strongest possible gravitational fields. So, spectral and temporal properties of a black hole should carry signatures of photon bending. Spectra of black hole candidates are primarily generated from a Keplerian disk (black-body) and a Compton cloud which is located in the inner region in the form of CENBOL (CT95). The physical parameters (accretion rates, shock location, shock strength, mass) of accretion process are extracted from the fitting of Two Component Advective Flow (TCAF) model (see Debnath et al. 2014; Mondal et al. 2014; Jana et al. 2016; Molla et al. 2016; Chatterjee et al. 2016). So, imaging a black hole surroundings would necessarily mean imaging of the Keplerian disk along with the CENBOL. Ours is the first paper, which creates a composite image of both the components, namely, the Keplerian and the sub-Keplerian components which are accreting at different accretion rates after addition of realistic physical processes. We ignore the pre-shock region of sub-Keplerian component since it is optically very thin and concentrate only on the centrifugal barrier region which 
primarily does the inverse Comptonization to produce the power-law component. We ignored the radial velocity inside this barrier since the flow is highly subsonic till it reaches the inner sonic point located between the marginally bound and marginally stable orbits. We also present the corresponding spectra and images for various flow parameters. We find that multi-color black-body part of a spectrum becomes flat at higher inclination angles. The number of photons in higher energy bin $(10-100 \mathrm{keV})$ increases with inclination angle. This is a clear effect of the focussing due to photon-bending. The spectra is harder at high inclination angles.

Our work has, for the first time, produced the images and also realistic spectra from a two component advective flow around a non-rotating black hole. Due to intrinsic nature of our Monte-Carlo simulation, we can also not only study the effects of bending on the timing properties, but also determine the time and phase lag properties. Similarly effects of spin would be very important and we would like to identify those features which are especially on spin dependent. This is outside the scope of this paper and would be reported elsewhere.

\section{ACKNOWLEDGEMENTS}

The work of AC is supported by Ministry of Earth Science (MoES), India.

\section{REFERENCES}

Abramowitz M., Stegun I. A., 1964, Handbook of Mathematical Functions, New York

Abramowicz M. A., Jaroszynski, M., Sikora M., 1978, A\&A, 63, 221

Armitage P. J., Reynolds C. S., 2003, MNRAS, 341, 1041

Bromley B. C., Melia F., Liu, S., 2001, ApJ, 555, 83

Chakrabarti S. K., 2002, Accretion Process on a Black Hole, Allied Publishers

Chakrabarti S. K., 1985, ApJ, 288, 1

Chakrabarti S. K., 1985, ApJ, 288, 7

Chakrabarti S. K., Jin L., Arnett D. W., 1987, ApJ, 313, 674

Chakrabarti S. K., 1990, Theory of Transonic Astrophysical Flows, World Scientific, Singapore

Chakrabarti S. K., 1990, MNRAS, 245, 747

Chakrabarti S. K., 1991, MNRAS, 250, 7

Chakrabarti S. K., Titarchuk L. G., 1995, ApJ, 455, 623

Chatterjee D., Debnath D., Chakrabarti S. K., Mondal S., Jana A., 2016, ApJ (In Press)

Chandrasekhar S., 1985, The Mathematical Theory of Black Holes, Oxford University press

Cunningham C. T., Bardeen J. M., 1973, ApJ, 183, 237

Debnath D., Mondal S., Chakrabarti S. K., 2014, MNRAS, 440, 121

Debnath D., Mondal S., Chakrabarti S. K., 2015, MNRAS, 447, 1984

Debnath D., Molla A. A., Chakrabarti S. K., Mondal S., 2015, ApJ, 803, 59 


\section{Arka Chatterjee, Sandip K. Chakrabarti \& Himadri Ghosh}

Dexter J., Agol E., 2009, ApJ, 696, 1616

Fabian A. C., Iwasawa K., Reynolds C. S., Young A. J., 2000, PASP, 49, 159

Fuerst S. V., Wu K., 2004, A\&A, 424, 733

Fukue J., Yokoyama T., 1988, PASJ, 40, 15

Ghosh H., Chakrabarti S. K., Laurent P., IJMPD, 18, 2009, 1693.

Ghosh H., Garain S. K., Chakrabarti S. K., Laurent P., 2010, IJMPD, 19, 607

Ghosh H., Garain S. K., Giri K., Chakrabarti S. K., 2011, MNRAS, 416, 959

Garain S. K., Ghosh H., Chakrabarti S. K., 2012, ApJ, 758, 114

Garain S. K., Ghosh H., Chakrabarti S. K., 2014, MNRAS, 437, 1329

Johnson M. D. et al., 2015, Science, 350, 6265, 1242

Jana A., Debnath D., Chakrabarti S. K., Mondal S., Molla A., A., 2016, ApJ, 819,107

Karas V., Vokrouhlicky D., Polnarev A. G., 1992, MNRAS, 259, 569

Kozlowski M., Jaroszynski, M., Abramowicz M. A., Jaroszynski, M., 1978, A\&A, 63, 209

Luminet J. P., 1979, A\&A, 75, 228

Laurent P., Titarchuk L. G., 1999, ApJ, 511, 289

Marck J. A., 1995, Classical and Quantum Gravity, 13, 393

Müller T., Frauendiener J., 2012, Eur. J. Phys, 33, 955

Molteni D., Lanzafame G., Chakrabarti S. K., 1994, ApJ, 425, 161

Mondal S., Debnath D., Chakrabarti S. K., 2014, ApJ, 786, 4

Molla A. A., Debnath D., Mondal S., Chakrabarti S. K., Jana, A., 2016, ApJ, (In Press)

Paczysky B., Wiita P. J., 1980, A\&A, 88, 23

Page D. N., and Thorne K. S., 1974, Astrophys. J., 191, 499

Pal P. S., Chakrabarti S. K., Nandi A., 2011, IJMPD, 20, 228

Pal P. S., Chakrabarti S. K., Nandi A., 2013, AdSpR, 52, 720

Pineault S., Roeder R. C., 1977, Astrophys. J., 212, 541

Pozdnyakov A., Sobol I. M., Sunyaev R. A., 1983, Astrophys. Space Sci. Rev., 2, 189

Rees M. J., Begelman M. C., Blandford R. D., Phinney, E. S., 1982, Nature, 295, 17

Schnittman J. D., Krolik J. H, ApJ, 2009, 712, 908

Shakura N. I., Sunyaev R. A., 1973, A\&A, 24, 337

Shapiro S. L., Teukolsky S. A., Black holes, White Dwarfs, and Neutron stars, 1983, Wiley-VCH, New York

Sunyaev R. A., Titarchuk L. G., 1985, A\&A, 143, 374

Viergutz S. U., Image generation in Kerr geometry, 1992, Astron. Astrophysics, 272, 355

Vincent F. H., Paumard T., Gourgoulhon E., Perrin G., 2011, Classical and Quantum Gravity 28, 225011

Weinberg S., 1972, Gravitation and Cosmology, John Wiley \& Sons, UK

Yang X., Wang, J., 2013, ApJS, 207, 6

Younsi Z., Wu k., Fuerst S. V., 2012, A\&A, 545, 13

Zeldovich Ya. B., Novikov I. D., 1971, Relativistic Astrophysics, University of Chicago Press, Chicago 